\title{
An estimator for the new bivariate copula
}

\begin{abstract}
Copula modelling is becoming more popular in modelling dependence multivariate distributions and various copulas have been suggested throughout the literature. In a previous study, we had proposed a new bivariate copula based on Rüschendorf method and had studied some properties of that copula. Therefore, in this paper, we propose an estimator for the new bivariate copula based on Kendallô Ü The bias and the asymptotic variance of the estimator are also given in this paper where a simulation study was conducted to verify these results. Hence, we find the proposed estimator based on Kendallô Üto be a suitable estimator for the new bivariate copula and this study adds on to contribute to the literature of the bivariate copula.
\end{abstract}

Keyword: Bivariate copula; Copula; Estimator 